\title{
A fixed-point anomaly in the plane
}

\author{
by
}

Charles L. Hagopian (Sacramento, CA) and

Janusz R. Prajs (Sacramento, CA, and Opole)

\begin{abstract}
We define an unusual continuum $M$ with the fixed-point property in the plane $\mathbb{R}^{2}$. There is a disk $D$ in $\mathbb{R}^{2}$ such that $M \cap D$ is an arc and $M \cup D$ does not have the fixed-point property. This example answers a question of R. H. Bing. The continuum $M$ is a countable union of arcs.
\end{abstract}

1. Introduction. It is an elementary fact that if two spaces have the fixed-point property, then so does their one-point union ([1, Theorem 6]). From the basic theory of retracts we know that all compact metric absolute retracts (AR's) have the fixed-point property, and if $X, Y$ and $X \cap Y$ are AR's, then so is $X \cup Y$ ([2, Theorem 6.1, p. 90]). Consequently, $X \cup Y$ has the fixed-point property. Instead of being an AR, if one of the spaces, say $X$, is only assumed to have the fixed-point property, must $X \cup Y$ still have the fixed-point property? The general answer to this question is no, even if we assume $X \cap Y$ and $Y$ to be as simple AR's as an arc and a disk, respectively. Indeed, in 1967, R. J. Knill [6] defined a 2-dimensional continuum $B$ with the fixed-point property and a disk $D$ such that $B \cap D$ is an arc and $B \cup D$ admits a fixed-point-free map. Also in 1967, W. Lopez [7] constructed a 17-dimensional polyhedron and R. H. Bing ([1, Theorems 14 and 15]) constructed a nonplanar 1-dimensional continuum with the properties of $B$.

In this paper, we present a negative answer to the following fundamental question that Bing asked in 1969.

Question 1 (Bing [1, Question 7]). If $C$ is a plane continuum with the fixed-point property and $D$ is a disk that intersects $C$ in an arc, must $C \cup D$ have the fixed-point property?

2000 Mathematics Subject Classification: 54F15, 54H25.

Key words and phrases: fixed-point property, folded spiral, plane continua, polar spiral, hereditarily decomposable continua.

The second author was supported in part by NSF-DMS-0405374.

The authors gratefully acknowledge several helpful conversations with Marcus Marsh. 
We actually show that even when $C \cup D$ is planar, it may fail to have the fixed-point property, thus answering a related question of V. Klee and S. Wagon [5, p. 149]. Our original example was much more complex than the one presented here. Several more months of study led to the present example, which is relatively simple. It has only four complementary domains in the plane. It is a countable union of arcs, has only five arc components and one ramification point (of order three).

In our construction we employ the concepts of polar and folded spirals in the plane. Planar spirals were used in other examples in fixed-point theory (for an early example see [12]). The results involving polar and folded spirals presented here should provide more precise tools for future constructions.

In this paper the term map means a continuous function. A continuum means a nonempty compact connected metric space. A space $X$ has the fixed-point property if each map $f: X \rightarrow X$ has a fixed point, i.e. a point $x \in X$ such that $f(x)=x$. A continuum is decomposable if it is the union of two proper subcontinua. A continuum is hereditarily decomposable if each of its nondegenerate subcontinua is decomposable. An acyclic hereditarily decomposable continuum is called a $\lambda$-dendroid. A set $X$ in the plane $\mathbb{R}^{2}$ is radially convex if for every point $x \in X$ the straight line segment from the origin to $x$ is contained in $X$. If $X \subset \mathbb{R}^{2}$ is a continuum such that $\mathbb{R}^{2} \backslash X$ is connected, then we say that $X$ is a nonseparating plane continuum.

2. Polar and folded spirals. We begin with some general observations about spirals in the plane.

Definition 1. A polar spiral is an embedding $s:[a, \infty) \rightarrow \mathbb{R}^{2}$ defined by a polar formula $s(t)=(\phi(t), t)$, where $a \in \mathbb{R}$ and $\phi$ is a nonnegative continuous function.

Definition 2. Let $K$ be a continuum in $\mathbb{R}^{2}$. Every polar spiral $s(t)$ in $\mathbb{R}^{2} \backslash K$ such that $\mathrm{Cl}(s([a, \infty))) \backslash s([a, \infty))=K$ is called a polar spiral limiting on $K$.

OBSERVATION 2.1. Let $p$ and $q$ be points of $\mathbb{R}^{2}$ such that $q$ does not belong to the radial line $O_{p}$ that starts at the origin and passes through $p$. Then for each $t>1$ we have $d\left(q, O_{p}\right)<d\left(t q, O_{p}\right)$.

Using this observation one can prove the following.

OBSERVATION 2.2. Let $F$ be a radially convex continuum in $\mathbb{R}^{2}$. For each pair of positive numbers $t$ and $\varepsilon$ there is a unique point $p(\varepsilon, t)$ such that $t$ is the $\theta$-coordinate of $p(\varepsilon, t)$ and $d(p(\varepsilon, t), F)=\varepsilon$. Moreover, the function $(t, \varepsilon) \mapsto p(t, \varepsilon)$ is continuous. 
Observation 2.3. For every radially convex continuum $F$ in $\mathbb{R}^{2}$ and every $a \in \mathbb{R}$, the formula $s(t)=p\left(e^{-t}, t\right)$ for $t \geq a$ defines a polar spiral limiting on $\operatorname{Bd}(F)$.

Observation 2.3 leads to one implication of the following proposition. The proof of the other implication is left to the reader.

Proposition 2.4. For every nonseparating plane continuum $F$, there exists a polar spiral in $\mathbb{R}^{2} \backslash F$ limiting on $\operatorname{Bd}(F)$ if and only if $F$ is radially convex.

Proposition 2.5. Let $F$ be a nonseparating plane continuum invariant with respect to the rotation $R_{\alpha}$ about the origin, $R_{\alpha}(r, \theta)=(r, \theta+\alpha)$, and let $s(t)$ be a polar spiral limiting on $\operatorname{Bd}(F)$. Then the map $s(t) \mapsto s(t+\alpha)$ defines a continuous extension of $R_{\alpha} \mid \operatorname{Bd}(F)$ to the set $\operatorname{Bd}(F) \cup s([0, \infty))$ if and only if

$$
\lim _{t \rightarrow \infty}(|s(t)|-|s(t+\alpha)|)=0 .
$$

Proof. Suppose that $s(t) \mapsto s(t+\alpha)$ continuously extends $R_{\alpha} \mid \operatorname{Bd}(F)$,

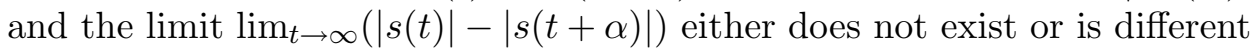
from 0 . Then there are positive numbers $t_{n}$ diverging to $\infty$ and $\varepsilon>0$ such that $\left|\left(\left|s\left(t_{n}\right)\right|-\left|s\left(t_{n}+\alpha\right)\right|\right)\right|>\varepsilon$ for each $n$. Since $s(t)$ limits on a compact set $\operatorname{Bd}(F)$, without loss of generality we may assume the sequence $\left\{s\left(t_{n}\right)\right\}$ converges to a point $p \in \operatorname{Bd}(F)$. By the continuity of the extended map we have $\lim _{n \rightarrow \infty} s\left(t_{n}+\alpha\right)=R_{\alpha}(p)$. Moreover, $\left|R_{\alpha}(p)\right|=|p|$. Therefore

$$
\lim _{n \rightarrow \infty}\left|s\left(t_{n}+\alpha\right)\right|=\left|R_{\alpha}(p)\right|=|p|=\lim _{n \rightarrow \infty}\left|s\left(t_{n}\right)\right| .
$$

Thus $\left|\left(\left|s\left(t_{n}+\alpha\right)\right|-\left|s\left(t_{n}\right)\right|\right)\right|<\varepsilon$ for almost all $n$, a contradiction.

Suppose that $(*)$ is satisfied. To prove that $s(t) \mapsto s(t+\alpha)$ continuously extends $R_{\alpha} \mid \operatorname{Bd}(F)$ it suffices to show that for each $p \in \operatorname{Bd}(F)$, if $\lim _{n \rightarrow \infty} s\left(t_{n}\right)=p$, then $\lim _{n \rightarrow \infty} s\left(t_{n}+\alpha\right)=R_{\alpha}(p)$. Fix a point $p=\left(|p|, t_{0}\right) \in$ $\operatorname{Bd}(F)$, and take any $t_{n} \in[0, \infty)$ satisfying $\lim _{n \rightarrow \infty} s\left(t_{n}\right)=p$. Observe that $\lim _{n \rightarrow \infty} t_{n}=\infty$. Hence $\lim _{n \rightarrow \infty}\left|s\left(t_{n}\right)\right|=|p|$, and $\lim _{n \rightarrow \infty}\left|s\left(t_{n}+\alpha\right)\right|=|p|$ by $(*)$. If $|p|=0$, then $p=(0,0)=R_{\alpha}(p)$, and $\lim _{n \rightarrow \infty}\left|s\left(t_{n}+\alpha\right)\right|=$ $\lim _{n \rightarrow \infty}\left|s\left(t_{n}\right)\right|=p=R_{\alpha}(p)$. Thus the conclusion holds. Suppose $|p|>0$. Since $|p|=\left|R_{\alpha}(p)\right|$ and the numbers $t_{n}$ converge to $t_{0}$ in the sense of the pseudo-distance $\varrho\left(t_{n}, t_{0}\right)=\min \left\{\left|t_{n}+2 k \pi-t_{0}\right|: k\right.$ is an integer $\}$, we have $\lim _{n \rightarrow \infty} s\left(t_{n}+\alpha\right)=\left(|p|, t_{0}+\alpha\right)=R_{\alpha}(p)$.

Let $F$ be a compact subset of the plane, and $G(F)$ be the group of rotations $R_{\alpha}$ of the plane about the origin such that $R_{\alpha}(F)=F$. Since every compact group of rotations is either discrete or homeomorphic to a circle, we have the following known result. 
Proposition 2.6. For each compact subset $F$ of the plane either $F$ is invariant with respect to every rotation about the origin, or there exists a minimal positive number $\alpha_{0}$ such that $G(F)=\left\{R_{k \alpha_{0}}: k \in \mathbb{Z}\right\}$. In particular, in the latter case $2 \pi$ is a multiple of $\alpha_{0}$.

OBSERVATION 2.7. Let $F$ be a nonseparating plane continuum invariant with respect to every rotation about the origin, and $s(t)$ be a polar spiral limiting on $\operatorname{Bd}(F)$. Then $F$ is either a point (the origin), or a disk, and for each number $\alpha$ the map $s(t) \mapsto s(t+\alpha)$ continuously extends the rotation $R_{\alpha} \mid F$.

If $\operatorname{Bd}(F)$ is not locally connected, one can prove that there are polar spirals limiting on $\mathrm{Bd}(F)$ that do not admit similar extensions of rotations. In the next proposition we prove that, nevertheless, such polar spirals can be replaced by ones admitting such an extension.

Proposition 2.8. Let $F$ be a nonseparating plane continuum invariant with respect to the rotation $R_{\alpha}$. If there exists a polar spiral $s(t)$ limiting on $\operatorname{Bd}(F)$, then there exists a polar spiral $s_{1}:[0, \infty) \rightarrow \mathbb{R}^{2}$ limiting on $\operatorname{Bd}(F)$ such that the map $s_{1}(t) \mapsto s_{1}(t+\alpha)$ continuously extends the rotation $R_{\alpha} \mid F$.

Proof. According to Proposition 2.4 the set $F$ is radially convex. Without loss of generality we may assume that $s(t)$ is the spiral $s(t)=p\left(e^{-t}, t\right)$, for $t \geq 0$, defined in Observation 2.3. If $F$ is a disk or a point, then $s_{1}=s$ satisfies the conclusion by Observation 3.4.

Suppose $F$ is neither a disk nor a point. It suffices to show the conclusion in the case $\alpha=\alpha_{0}$, where $\alpha_{0}$ is the minimal positive angle such that $R_{\alpha_{0}}$ belongs to $G(F)$ (see Proposition 2.6). Note that by the invariance of $F$ with respect to $R_{\alpha}$, and by the definition of $s(t)$, which also uses Observation 2.2, we have $|s(t+\alpha)|<|s(t)|$. Define a function $\phi: \mathbb{R} \rightarrow \mathbb{R}_{+}$by letting $\phi(t)=$ $|p(1, t)|$ (see Observation 2.3) for $t \leq 0$, and recursively,

$$
\phi(t)=\max \left\{|s(t)|, \phi(t-\alpha)-\frac{t}{t^{2}+1}\right\} \quad \text { for } t>0 .
$$

We prove that the spiral $s_{1}(t)=(\phi(t), t)$ for $t \geq 0$ satisfies the conclusion.

First, note that

$$
|s(t+\alpha)|<|s(t)| \leq \phi(t) \quad \text { and } \quad \phi(t)-\frac{t+\alpha}{(t+\alpha)^{2}+1}<\phi(t) .
$$

Therefore

$$
\phi(t+\alpha)=\max \left\{|s(t+\alpha)|, \phi(t)-\frac{t+\alpha}{(t+\alpha)^{2}+1}\right\}<\phi(t) \quad \text { for all } t \geq 0 .
$$


In particular, since $2 \pi$ is a multiple of $\alpha$ (see Proposition 2.6), we have $\left|s_{1}(t+2 \pi)\right|=\phi(t+2 \pi)<\phi(t)=\left|s_{1}(t)\right|$ for $t \geq 0$, and thus $s_{1}$ has no self-intersections. Observe that for every $t \geq 0$ we have $|s(t)| \leq\left|s_{1}(t)\right|$ by the definition of $s_{1}$.

We also prove that $\left|s_{1}(t+2 k \pi)\right| \leq|s(t)|$ for infinitely many positive integers $k$. Indeed, otherwise we would have $\phi(t+2 k \pi)=\left|s_{1}(t+2 k \pi)\right|>$ $|s(t)|>|s(t+2 k \pi)|$ for $k \in\left\{k_{0}, k_{0}+1, \ldots\right\}$, where $k_{0}$ is some positive integer. Note that $(t+2 k \pi-\alpha)-(t+2(k-1) \pi)=2 \pi-\alpha$ is a multiple of $\alpha$. Therefore

$$
\begin{aligned}
\phi(t+2 k \pi) & =\phi(t+2 k \pi-\alpha)-\frac{t+2 k \pi}{(t+2 k \pi)^{2}+1} \\
& \leq \phi(t+2(k-1) \pi)-\frac{t+2 k \pi}{(t+2 k \pi)^{2}+1}
\end{aligned}
$$

and thus

$$
\phi(t+2(k-1) \pi)-\phi(t+2 k \pi) \geq \frac{t+2 k \pi}{(t+2 k \pi)^{2}+1} .
$$

Consequently,

$$
\phi\left(t+2 k_{0} \pi\right)-\phi(t+2 k \pi) \geq \sum_{l=k_{0}+1}^{k} \frac{t+2 l \pi}{(t+2 l \pi)^{2}+1} \rightarrow \infty \quad \text { as } k \rightarrow \infty .
$$

Hence $\phi(t+2 k \pi)$ would be negative for some $k$, which is impossible by the definition of $\phi$.

Using the inequalities $|s(t+2 k \pi)| \leq\left|s_{1}(t+2 k \pi)\right| \leq|s(t)|$ (for infinitely many $k$ 's) and $\left|s_{1}(t+2 \pi)\right|<\left|s_{1}(t)\right|$ (for all $t$ ), one can prove that, since $s(t)$ limits on $\operatorname{Bd}(F)$, so does $s_{1}(t)$. By the definitions of $\phi$ and $s_{1}$ we also have $\left|s_{1}(t)\right|-\left|s_{1}(t+\alpha)\right| \leq t /\left(t^{2}+1\right)$. Hence the function $s_{1}(t) \mapsto s_{1}(t+\alpha)$ continuously extends the rotation $R_{\alpha} \mid F$ by Proposition 2.5. The proof is complete.

Proposition 2.9. Let $F$ be a nonseparating plane continuum invariant with respect to a rotation $R_{\alpha}$, and $s_{1}(t)$ and $s_{2}(t)$ be two disjoint polar spirals limiting on $\operatorname{Bd}(F)$. Then the map $s_{1}(t) \mapsto s_{1}(t+\alpha)$ continuously extends $R_{\alpha} \mid F$ if and only if $s_{2}(t) \mapsto s_{2}(t+\alpha)$ does.

Proof. Suppose that $s_{1}(t) \mapsto s_{1}(t+\alpha)$ continuously extends $R_{\alpha} \mid F$ (the proof of the other case is similar). Then, for some $t_{0}$, there is an integer $m$ (maybe negative) such that $s_{2}\left(t_{0}\right)$ belongs to the straight line segment from $s_{1}\left(t_{0}+2 m \pi\right)$ to $s_{1}\left(t_{0}+2 m \pi+2 \pi\right)$. Each triple of points $s_{2}(t), s_{1}(t+2 m \pi)$ and $s_{1}(t+2 m \pi+2 \pi)$ have common polar coordinate $\theta$, and thus they are collinear. Since $s_{2}\left(t_{0}\right)$ is between $s_{1}\left(t_{0}+2 m \pi\right)$ and $s_{1}\left(t_{0}+2 m \pi+2 \pi\right)$, the points $s_{2}(t), s_{1}(t+2 m \pi)$ and $s_{1}(t+2 m \pi+2 \pi)$ are collinear for each $t$, and $s_{1}$ and $s_{2}$ are disjoint, it follows that $s_{2}(t)$ belongs to the straight 
line segment from $s_{1}(t+2 m \pi)$ to $s_{1}(t+2 m \pi+2 \pi)$ for each $t>t_{0}$. Note that

$$
\lim _{t \rightarrow \infty} d\left(s_{1}(t+2 m \pi), s_{1}(t+2 m \pi+2 \pi)\right)=0
$$

because the spiral $s_{1}$ limits on $\operatorname{Bd}(F)$.

To prove the continuity of the extension $s_{2}(t) \mapsto s_{2}(t+\alpha)$ of $R_{\alpha} \mid F$, suppose $\lim s_{2}\left(t_{n}\right)=p$ for some sequence of numbers $t_{n}$ and some $p \in$ $\operatorname{Bd}(F)$. Since $s_{2}$ limits on $\operatorname{Bd}(F)$, we have $\lim t_{n}=\infty$. Therefore, for sufficiently large $n$ the point $s_{2}\left(t_{n}\right)$ belongs to the straight line segment from $s_{1}\left(t_{n}+2 m \pi\right)$ to $s_{1}\left(t_{n}+2 m \pi+2 \pi\right)$, and the point $s_{2}\left(t_{n}+\alpha\right)$ belongs to the straight line segment from $s_{1}\left(t_{n}+\alpha+2 m \pi\right)$ to $s_{1}\left(t_{n}+\alpha+2 m \pi+2 \pi\right)$. We also have

$$
\begin{aligned}
\lim d\left(s_{1}\left(t_{n}+2 m \pi\right), s_{1}\left(t_{n}+2 m \pi+2 \pi\right)\right) & \\
& =\lim d\left(s_{1}\left(t_{n}+\alpha+2 m \pi\right), s_{1}\left(t_{n}+\alpha+2 m \pi+2 \pi\right)\right)=0 .
\end{aligned}
$$

Consequently, $\lim s_{1}\left(t_{n}+2 m \pi\right)=\lim s_{1}\left(t_{n}+2 m \pi+2 \pi\right)=p$. By the continuity of the extension $s_{1}(t) \mapsto s_{1}(t+\alpha)$ we have

$$
\lim s_{1}\left(t_{n}+\alpha+2 m \pi\right)=\lim s_{1}\left(t_{n}+\alpha+2 m \pi+2 \pi\right)=R_{\alpha}(p) .
$$

Hence $\lim s_{2}\left(t_{n}+\alpha\right)=R_{\alpha}(p)$.

If we have a finite collection of mutually disjoint polar spirals limiting on the same set, then each arc in any of the spirals is free in the closure of the union of the spirals. Thus the spirals admit mutually disjoint neighborhoods in the plane that are also disjoint from the limiting set. This leads to the following observation.

OBSERVATION 2.10. Let $F$ be a nonseparating plane continuum and $s_{1}(t), \ldots, s_{k}(t)$ be mutually disjoint polar spirals limiting on $\operatorname{Bd}(F)$. Then there are polar spirals $\bar{s}_{1}(t), \ldots, \bar{s}_{k}(t)$ such that for every $t$ the straight line segments from $s_{i}(t+2 j \pi)$ to $\bar{s}_{i}(t+2 j \pi)$ are mutually disjoint for $i \in\{1, \ldots, k\}$ and $j \in\{0,1, \ldots\}$. (In particular, the spirals $s_{1}(t), \ldots, s_{k}(t)$, $\bar{s}_{1}(t), \ldots, \bar{s}_{k}(t)$ are mutually disjoint. $)$

Let $s_{i}(t)$ be one of the spirals $s_{1}(t), \ldots, s_{k}(t)$ as in Observation 2.10. Let $\bar{s}_{i}(t)$ be the corresponding spiral guaranteed by the observation. Then the formula $(1-u) \cdot s_{i}(t)+u \cdot \bar{s}_{i}(t)$, for $u \in[0,1]$, defines a homotopy from $s_{i}(t)$ to $\bar{s}_{i}(t)$ of mutually disjoint polar spirals limiting on $\operatorname{Bd}(F)$. Reparametrizing $u$ by the formula $w=u /(1-u)$ we obtain a continuous family of polar spirals $s_{i}(t, w)$ for $w \in[0, \infty)$ with $s_{i}(t)=s_{i}(t, 0)$. (The spiral $\bar{s}_{i}(t)$ would correspond to $s_{i}(t, \infty)$, though this notation will not be used.) Note that, in view of the previous observation, the spirals $s_{i}\left(t, w_{1}\right)$ and $s_{j}\left(t, w_{2}\right)$ are disjoint whenever either $i \neq j$ or $w_{1} \neq w_{2}$. 
Definition 3. For every continuous function $t:[0, \infty) \rightarrow[0, \infty)$ such that $\lim _{w \rightarrow \infty} t(w)=\infty$ the function $\widehat{s}_{i}:[0, \infty) \rightarrow \mathbb{R}^{2}$ defined by $\widehat{s}_{i}(w)=$ $s_{i}(t(w), w)$, where $s_{i}(t, w)$ is the continuous collection of polar spirals described above, is called a folded spiral following $s_{i}$ and limiting on $\operatorname{Bd}(F)$ (actual folds appear if $t(w)$ is not monotone).

Suppose $F$ is invariant with respect to a rotation $R_{\alpha}$ and the map $s_{i}(t) \mapsto s_{i}(t+\alpha)$ continuously extends $R_{\alpha} \mid F$. In the next proposition we discuss a sufficient condition implying that a folded spiral $\widehat{s}_{i}(w)$ following $s_{i}(t)$ admits a similar extension. To simplify notation we use $s(t), \bar{s}(t), s(t, w)$, and $\widehat{s}(w)=s(t(w), w)$ instead of $s_{i}(t), \bar{s}_{i}(t), s_{i}(t, w)$, and $\widehat{s}_{i}(w)=s_{i}(t(w), w)$, respectively.

Proposition 2.11. Let $F$ be a nonseparating plane continuum invariant with respect to a rotation $R_{\alpha}$, and $s(t)$ be a polar spiral limiting on $\operatorname{Bd}(F)$ such that the map $s(t) \mapsto s(t+\alpha)$ continuously extends the rotation $R_{\alpha} \mid F$. Let $\bar{s}(t)$ be the spiral guaranteed by Observation 2.10 (with, possibly, some other spirals $s_{j}(t)$ considered), and let $s(t, w)$ be a corresponding continuous family of spirals discussed above. Suppose that $\phi$ is a real continuous periodic function with period $\alpha$ such that $t(w)=w+\phi(w)$ is nonnegative for $w \geq 0$. Then the map $\widehat{s}(w)=s(t(w), w)$ is a folded spiral following $s(t)$ such that $\widehat{s}(w) \mapsto \widehat{s}(w+\alpha)$ continuously extends the rotation $R_{\alpha} \mid F$.

Proof. To prove that $\widehat{s}(w) \mapsto \widehat{s}(w+\alpha)$ continuously extends the rotation $R_{\alpha} \mid F$, suppose that $\lim \widehat{s}\left(w_{n}\right)=p$ for some sequence $w_{n}$ of numbers and some $p \in \operatorname{Bd}(F)$. Since $\widehat{s}(w)$ limits on $\operatorname{Bd}(F)$, it follows that $\lim w_{n}=\infty$. Therefore, by the definition we have $\lim t\left(w_{n}\right)=\infty$. Note that $\widehat{s}\left(w_{n}\right)$ belongs to the straight line segment from $s\left(t\left(w_{n}\right)\right)$ to $\bar{s}\left(t\left(w_{n}\right)\right)$ for each $n$. The spirals $s(t)$ and $\bar{s}(t)$ both limit on $\operatorname{Bd}(F)$ and $\lim t\left(w_{n}\right)=\infty$, which implies that $\lim d\left(s\left(t\left(w_{n}\right)\right), \bar{s}\left(t\left(w_{n}\right)\right)\right)=0$. Thus

$$
\lim s\left(t\left(w_{n}\right)\right)=\lim \bar{s}\left(t\left(w_{n}\right)\right)=\lim \widehat{s}\left(w_{n}\right)=p .
$$

Since $s(t) \mapsto s(t+\alpha)$ continuously extends $R_{\alpha} \mid F$, so does the map $\bar{s}(t) \mapsto$ $\bar{s}(t+\alpha)$ by Proposition 2.9. Consequently,

$$
\lim s\left(t\left(w_{n}\right)+\alpha\right)=\lim \bar{s}\left(t\left(w_{n}\right)+\alpha\right)=R_{\alpha}(p) .
$$

Note that

$$
t\left(w_{n}+\alpha\right)=\left(w_{n}+\alpha\right)+\phi\left(w_{n}+\alpha\right)=w_{n}+\alpha+\phi\left(w_{n}\right)=t\left(w_{n}\right)+\alpha,
$$

and thus $\widehat{s}\left(w_{n}+\alpha\right)$ belongs to the straight line segment from $s\left(t\left(w_{n}\right)+\alpha\right)$ to $\bar{s}\left(t\left(w_{n}\right)+\alpha\right)$ for each $n$. Since the limit of the lengths of these segments is 0 , we have $\lim \widehat{s}\left(w_{n}+\alpha\right)=R_{\alpha}(p)$. The proof is complete.

3. The construction. We begin with a construction of a double Warsaw circle with three limiting spirals. The spirals admit extensions 
of the 180-degree rotation of the Warsaw circle. Such extensions were previously discussed in [9], [4], and [10]. Here the construction of spirals and their extensions is based on the theory developed in the previous section.

Define the polar equation

$$
r(\theta)=\frac{3}{2}+\frac{1}{2} \cos \left(\tan \frac{\theta}{2}\right) \quad \text { for } 0 \leq \theta<\pi,
$$

and $r(\theta)=r(\theta-\pi)$ for $\pi \leq \theta<2 \pi$. Let $W$ be the closure of the graph of $r(\theta)$ (the double Warsaw circle). The union of $W$ and the bounded complementary domain of $W$ is radially convex with respect to the origin. Note that the union is invariant with respect to the rotation $R_{\pi}$, and $W$ is its boundary in the plane. Let $W_{A}$ and $W_{B}$ be the two arc components of $W$, where the $\theta$-coordinates of the points in $W_{A}$ belong to $[0, \pi)$, and of those in $W_{B}$ to $[\pi, 2 \pi)$.

According to Observation 2.3 and Proposition 2.8 there is a polar spiral $s_{0}(t)$ in the unbounded complementary domain of $W$, limiting on $W$, such that the map $s_{0}(t) \mapsto s_{0}(t+\pi)$ defines a continuous extension of $R_{\pi} \mid W$ to the set $W \cup s_{0}([0, \infty))$. Applying Observation 2.10 twice, we define, in the unbounded complementary domain of $W$, two other polar spirals $s_{1}(t)$ and $s_{2}(t)$ limiting on $W$ such that $s_{0}, s_{1}$ and $s_{2}$ are mutually disjoint. By Proposition 2.9 the maps $s_{1}(t) \mapsto s_{1}(t+\pi)$ and $s_{2}(t) \mapsto s_{2}(t+\pi)$ continuously extend the rotation $R_{\pi} \mid W$.

Let

$$
\phi_{0}(w)=7 \pi \sin ^{2} w, \quad \phi_{1}(w)=3 \pi \sin ^{2} w, \quad \phi_{2}(w)=3 \pi \sin ^{2} w .
$$

Note that $\phi_{0}, \phi_{1}$ and $\phi_{2}$ are continuous and periodic with period $\pi$. Let $\widehat{s}_{0}(w), \widehat{s}_{1}(w), \widehat{s}_{2}(w)$ be mutually disjoint folded spirals following $s_{0}(t), s_{1}(t)$, $s_{2}(t)$, respectively, with the corresponding folding functions $t_{0}(w)=w+$ $\phi_{0}(w), t_{1}(w)=w+\phi_{1}(w)$ and $t_{2}(w)=w+\phi_{2}(w)$ (see Definition 3). Note that, according to Proposition 2.11, the maps $\widehat{s}_{0}(w) \mapsto \widehat{s}_{0}(w+\pi)$, $\widehat{s}_{1}(w) \mapsto \widehat{s}_{1}(w+\pi), \widehat{s}_{2}(w) \mapsto \widehat{s}_{2}(w+\pi)$ continuously extend the rotation $R_{\pi} \mid W$. Let

$$
\begin{gathered}
\widehat{S}_{0}=\widehat{s}_{0}([0, \infty)), \quad \widehat{S}_{1}=\widehat{s}_{1}([0, \infty)), \\
\widehat{S}_{2}=\widehat{s}_{2}([0, \infty)), \quad \Omega_{0}=W \cup \widehat{S}_{0} \cup \widehat{S}_{1} \cup \widehat{S}_{2} .
\end{gathered}
$$

We need the following lemma to study the mapping properties of $\Omega_{0}$.

LEMMA 3.1. Let

$$
\begin{aligned}
& g_{1}(x)=7 \sin ^{2} x-z(x)-3 \sin ^{2}(x+\pi z(x)), \\
& g_{2}(x)=3 \sin ^{2} x-z(x)-7 \sin ^{2}(x+\pi z(x)),
\end{aligned}
$$


for $x \in[0, \infty)$, where $z(x)$ is a real continuous function. Define

$$
\begin{aligned}
& \psi_{1}(x)=e^{\pi g_{1}(x) i}=\cos \left(\pi g_{1}(x)\right)+i \sin \left(\pi g_{1}(x)\right), \\
& \psi_{2}(x)=e^{\pi g_{2}(x) i}=\cos \left(\pi g_{2}(x)\right)+i \sin \left(\pi g_{2}(x)\right) .
\end{aligned}
$$

Then for every number $M$ there are numbers $\tilde{x}_{j}>M, j \in\{1,2\}$, such that $\psi_{j}\left(\tilde{x}_{j}\right)=-1$.

Proof. Suppose that there are $M$ and $j$ such that $\psi_{j}(x) \neq-1$ for all $x>M$. Then, for some integer $k$, we have $(2 k-1) \pi<g_{j}(x) \pi<(2 k+1) \pi$ whenever $x>M$. Consequently, $\left|g_{j}(b)-g_{j}(a)\right|<2$ for all $a, b>M$. In particular $g_{j}$ is a bounded function.

Case 1 . Suppose $j=1$. Let $m$ be an integer such that $m \pi>M$. We have

$$
\begin{aligned}
2> & g_{1}(m \pi+\pi / 2)-g_{1}(m \pi) \\
= & 7 \sin ^{2}(m \pi+\pi / 2)-z(m \pi+\pi / 2) \\
& -3 \sin ^{2}(m \pi+\pi / 2+\pi z(m \pi+\pi / 2)) \\
& -\left[7 \sin ^{2}(m \pi)-z(m \pi)-3 \sin ^{2}(m \pi+\pi z(m \pi))\right] \\
= & 7-[z(m \pi+\pi / 2)-z(m \pi)] \\
& -3\left[\sin ^{2}(m \pi+\pi / 2+\pi z(m \pi+\pi / 2))-\sin ^{2}(m \pi+\pi z(m \pi))\right] \\
\geq & 4-[z(m \pi+\pi / 2)-z(m \pi)]
\end{aligned}
$$

Therefore $z(m \pi+\pi / 2)-z(m \pi)>2$ and

$$
[(m \pi+\pi / 2)+\pi z(m \pi+\pi / 2)]-[m \pi+\pi z(m \pi)]>\pi / 2+2 \pi>2 \pi .
$$

Since the function $x+\pi z(x)$ is continuous, there are numbers $a, b$ such that $m \pi \leq a<b \leq m \pi+\pi / 2, a+\pi z(a)=l \pi-\pi / 2$ and $b+\pi z(b)=l \pi$ for some integer $l$. So $(b+\pi z(b))-(a+\pi z(a))=\pi / 2$, and thus $z(b)-z(a)<1 / 2$. Consequently,

$$
\begin{aligned}
g_{1}(b)-g_{1}(a)= & 7\left(\sin ^{2} b-\sin ^{2} a\right)-[z(b)-z(a)] \\
& -3\left[\sin ^{2}(\pi l)-\sin ^{2}(\pi l-\pi / 2)\right] \\
> & 7\left(\sin ^{2} b-\sin ^{2} a\right)-1 / 2-(-3) \\
= & 7\left(\sin ^{2} b-\sin ^{2} a\right)+5 / 2 .
\end{aligned}
$$

The condition $m \pi \leq a<b \leq m \pi+\pi / 2$ implies $\sin ^{2} a<\sin ^{2} b$. Hence $g_{1}(b)-g_{1}(a)>5 / 2>2$, a contradiction.

CASE 2. Suppose $j=2$. Since $g_{2}$ and $\sin ^{2}$ are bounded functions, it follows that so is $z$. Therefore $\lim _{x \rightarrow \infty}(x+\pi z(x))=\infty$. By the continuity of the function $x+\pi z(x)$ there are numbers $a, b$ such that $M<a<b$, 
$a+\pi z(a)=l \pi-\pi / 2$ and $b+\pi z(b)=l \pi$ for some integer $l$. Similarly to the previous case, we conclude that $z(b)-z(a)<1 / 2$. Hence

$$
\begin{aligned}
g_{2}(b) & -g_{2}(a) \\
= & 3\left(\sin ^{2} b-\sin ^{2} a\right)-[z(b)-z(a)]-7\left[\sin ^{2}(b+\pi z(b))-\sin ^{2}(a+\pi z(a))\right] \\
= & 3\left(\sin ^{2} b-\sin ^{2} a\right)-[z(b)-z(a)]-7\left[\sin ^{2}(l \pi)-\sin ^{2}(l \pi-\pi / 2)\right] \\
= & 7+3\left(\sin ^{2} b-\sin ^{2} a\right)-[z(b)-z(a)] \\
& \geq 4-(z(b)-z(a))>4-1 / 2>2,
\end{aligned}
$$

a contradiction. The proof is complete.

The following property of $\Omega_{0}$ will be used in the study of the main example.

Property 3.2. Let $f: \Omega_{0} \rightarrow \Omega_{0}$ be a continuous function such that $f(W)=W$. Then $f\left(\widehat{S}_{0}\right) \subset \widehat{S}_{0}$ and $f\left(\widehat{S}_{1} \cup \widehat{S}_{2}\right) \subset \widehat{S}_{1} \cup \widehat{S}_{2}$.

Proof. Let $g$ be the rotation $R_{\pi} \mid W$ extended to $\Omega_{0}$ by the mappings $\widehat{s}_{k}(w) \mapsto \widehat{s}_{k}(w+\pi)$ for $k \in\{0,1,2\}$. Since continuous maps preserve arc connectedness, and $W_{A}$ and $W_{B}$ are all arc components of $W$ and $f(W)=W$, it follows that either $f\left(W_{A}\right)=W_{A}$ and $f\left(W_{B}\right)=W_{B}$, or $f\left(W_{A}\right)=W_{B}$ and $f\left(W_{B}\right)=W_{A}$. In the latter case we first replace $f$ with $f^{\prime}=g \circ f$, which satisfies the former condition, i.e., $f^{\prime}\left(W_{A}\right)=W_{A}$ and $f^{\prime}\left(W_{B}\right)=W_{B}$. Observe that if the property holds for $f^{\prime}$ (instead of $f$ ), then it holds for $f$ because $g\left(\widehat{S}_{k}\right) \subset \widehat{S}_{k}$ for $k \in\{0,1,2\}$. Therefore, without loss of generality, we assume that $f\left(W_{A}\right)=W_{A}$ and $f\left(W_{B}\right)=W_{B}$.

Observe that for each $k \in\{0,1,2\}$ there is a $j \in\{0,1,2\}$ such that $f\left(\widehat{S}_{k}\right) \subset \widehat{S}_{j}$. Indeed, otherwise $f\left(\widehat{S}_{k}\right) \subset W_{A}$ or $f\left(\widehat{S}_{k}\right) \subset W_{B}$. Since $\widehat{s}_{k}(w)$ limits on $W$, we would have $f(W) \subset \mathrm{Cl}\left(W_{A}\right) \neq W$ or $f(W) \subset \mathrm{Cl}\left(W_{B}\right) \neq W$, contrary to the assumption $f(W)=W$.

For each point $p \in \Omega_{0}$ let $\theta(p)$ be the $\theta$-coordinate of $p$ (though $\theta(p)$ is not unique), and let $\psi(p)=e^{[\theta(p)-\theta(f(p))] i}$. Note that $\psi$ is uniquely defined and continuous. Observe that $\psi(p)=-1$ is equivalent to the fact that $p$ and $f(p)$ are collinear with the origin and lie on the opposite sides of the origin. Since $f\left(W_{A}\right)=W_{A}$ and $f\left(W_{B}\right)=W_{B}$, we have $\psi(p) \neq-1$ for each $p \in W$.

Note that for each point $p=\widehat{s}_{j}(w) \in \widehat{S}_{j}$ the number $\theta(p)$ can be represented as $w+\phi_{j}(w)$ for $j \in\{0,1,2\}$. Suppose $f\left(\widehat{S}_{j}\right) \subset \widehat{S}_{k}$. Since $w \mapsto \widehat{s}_{m}(w)$ is an embedding for $m \in\{0,1,2\}$, we define $h_{k, j}:[0, \infty) \rightarrow[0, \infty)$ by $h_{k, j}(w)=\widehat{s}_{j}^{-1}\left(f\left(\widehat{s}_{k}(w)\right)\right)$ and note that $h_{k, j}$ is continuous.

Claim 1. $f\left(\widehat{S}_{0}\right) \subset \widehat{S}_{0}$. 
Indeed, suppose $f\left(\widehat{S}_{0}\right) \subset \widehat{S}_{j}$ for some $j \in\{1,2\}$. Assume $j=1$ (the other case is similar). For each point $p=\widehat{s}_{0}(w) \in \widehat{S}_{0}$ we can define

$$
\begin{aligned}
\theta(p) & =w+\phi_{0}(w)=w+7 \pi \sin ^{2} w \\
\theta(f(p)) & =h_{0,1}(w)+\phi_{1}\left(h_{0,1}(w)\right)=h_{0,1}(w)+3 \pi \sin ^{2}\left(h_{0,1}(w)\right) .
\end{aligned}
$$

Let $z_{1}(w)=\left(h_{0,1}(w)-w\right) / \pi$. Then

$$
\begin{aligned}
\theta(p)-\theta(f(p)) & =w+7 \pi \sin ^{2} w-\left[h_{0,1}(w)+3 \pi \sin ^{2}\left(h_{0,1}(w)\right)\right] \\
& =7 \pi \sin ^{2} w-\pi z_{1}(w)-3 \pi \sin ^{2}\left(w+\pi z_{1}(w)\right) \\
& =\pi\left[7 \sin ^{2} w-z_{1}(w)-3 \sin ^{2}\left(w+\pi z_{1}(w)\right)\right] .
\end{aligned}
$$

According to Lemma 3.1 (apply the function $g_{1}$ ), there are arbitrarily large numbers $w_{n}$ with corresponding points $p_{n}=\widehat{s}_{0}\left(w_{n}\right) \in \widehat{S}_{0}$ such that

$$
\psi\left(p_{n}\right)=e^{\left[\theta\left(p_{n}\right)-\theta\left(f\left(p_{n}\right)\right)\right] i}=e^{\pi\left[7 \sin ^{2} w_{n}-z_{1}\left(w_{n}\right)-3 \sin ^{2}\left(w_{n}+\pi z_{1}\left(w_{n}\right)\right)\right] i}=-1 .
$$

The spiral $\widehat{S}_{0}$ limits on $W$. Hence there is a point $p_{0} \in W$ such that $\psi\left(p_{0}\right)=-1$, a contradiction.

Claim 2. $f\left(\widehat{S}_{1} \cup \widehat{S}_{2}\right) \subset \widehat{S}_{1} \cup \widehat{S}_{2}$.

Indeed, to prove this claim it suffices to show $f\left(\widehat{S}_{1}\right) \not \subset \widehat{S}_{0}$ and $f\left(\widehat{S}_{2}\right) \not \subset \widehat{S}_{0}$. This can be done using a similar argument as in the proof of Claim 1 for $j=1$ or $j=2$ and $k=0$, and applying the function $g_{2}$ from Lemma 3.1. The details are left to the reader.

Let $T$ be a simple triod in the plane with the ramification point $r$ and one end point at $\widehat{s}_{0}(0)$ such that $T \cap \Omega_{0}=\left\{\widehat{s}_{0}(0)\right\}$. Denote the other end points of $T$ by $p_{1}$ and $p_{2}$. Choose a point $q$ in the arc in $T$ from $r$ to $p_{2}$ such that $r \neq q \neq p_{2}$, and a point $a$ in the arc in $T$ from $r$ to $q$ such that $r \neq a \neq q$. Additionally, we choose a point $b$ in the arc in $T$ from $r$ to $\widehat{s}_{0}(0)$ such that $r \neq b \neq \widehat{s}_{0}(0)$. In what follows we employ the notation $p_{1} r, p_{2} q$, $r q, r a$, etc., for arcs in $T$ having the corresponding end points. Let $L_{1}$ and $L_{2}$ be rays in the plane, i.e. homeomorphic copies of $[0, \infty)$, whose only end points are $\widehat{s}_{1}(0)$ and $\widehat{s}_{2}(0)$, respectively, such that

(1) each of the sets $\mathrm{Cl}\left(L_{1}\right)$ and $\mathrm{Cl}\left(L_{2}\right)$ is homeomorphic to the $\sin (1 / x)$ curve with the convergence arc $p_{1} r$ and $p_{2} q$, respectively;

(2) we have $L_{1} \cap L_{2}=\emptyset, L_{1} \cap\left(\Omega_{0} \cup T\right)=\left\{\widehat{s}_{1}(0)\right\}$ and $L_{2} \cap\left(\Omega_{0} \cup T\right)=$ $\left\{\widehat{s}_{2}(0)\right\}$.

The set $\Omega=\Omega_{0} \cup T \cup L_{1} \cup L_{2}$ is the main example of the paper. The figure below represents $\Omega$, though, in the part of the picture corresponding to $\Omega_{0}$, the folds of the spirals $\widehat{S}_{0}, \widehat{S}_{1}$ and $\widehat{S}_{2}$ are not shown. (Including the folds would heavily compromise the clarity of the picture. The reader can imagine that the folds are so tight that they disappear from view.) 


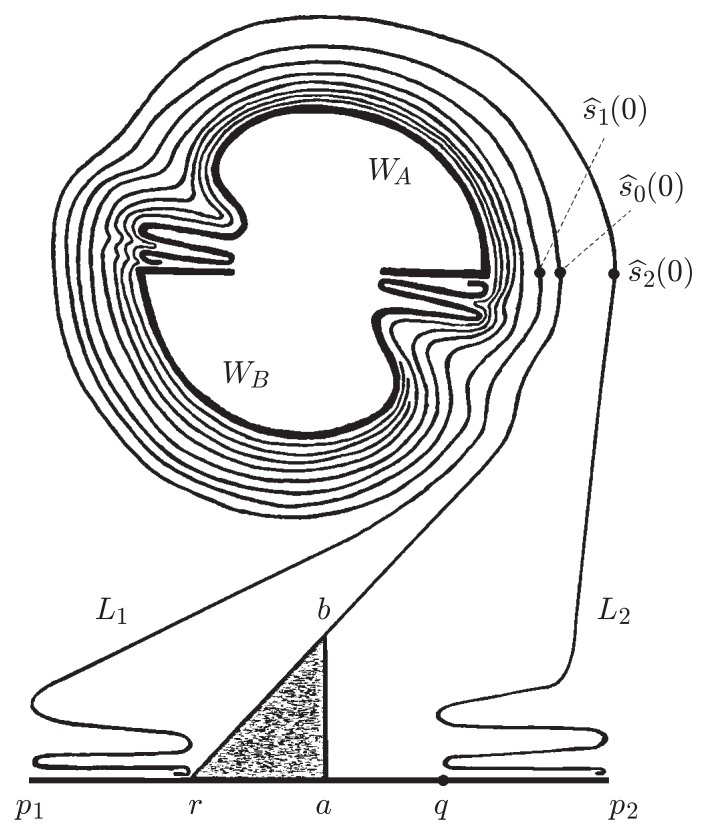

Observe that $\Omega$ is a hereditarily decomposable continuum in the plane. Define $S_{0}=\widehat{S}_{0} \cup T, S_{1}=\widehat{S}_{1} \cup L_{1}$ and $S_{2}=\widehat{S}_{2} \cup L_{2}$. Note that $W_{A}, W_{B}, S_{0}$, $S_{1}$ and $S_{2}$ are all the arc components of $\Omega$.

Property 3.3. The continuum $\Omega$ has the fixed-point property.

Proof. Suppose that $f: \Omega \rightarrow \Omega$ is a fixed-point-free map. First note that $f\left(S_{j}\right) \cap W=\emptyset$ for $j \in\{0,1,2\}$. Indeed, otherwise $f\left(S_{j}\right) \subset W_{A}$ or $f\left(S_{j}\right) \subset W_{B}$ by the continuity of $f$. Since $\widehat{S}_{j} \subset S_{j}$ limits on $W$, we would have either $f\left(\mathrm{Cl}\left(W_{A}\right)\right) \subset f(W) \subset \mathrm{Cl}\left(W_{A}\right)$ or $f\left(\mathrm{Cl}\left(W_{B}\right)\right) \subset f(W) \subset \mathrm{Cl}\left(W_{B}\right)$. Both sets $\mathrm{Cl}\left(W_{A}\right)$ and $\mathrm{Cl}\left(W_{B}\right)$ have the fixed-point property, and thus one of them would contain a fixed point of $f$, a contradiction.

Second, notice that $W \subset f(\Omega)$. Indeed, observe that every subcontinuum $K$ of $\Omega$ such that $W \backslash K \neq \emptyset$ does not separate the plane and is hereditarily decomposable. Therefore it is a $\lambda$-dendroid. All $\lambda$-dendroids have the fixedpoint property [8]. In the case $W \backslash f(\Omega) \neq \emptyset$, the image $f(\Omega)$ would have the fixed-point property, and thus it would contain a fixed point of $f$, a contradiction.

Since $f\left(S_{j}\right) \cap W=\emptyset$ for $j \in\{0,1,2\}$ and $W \subset f(\Omega)$, it follows that $f(W)=W$ and $f(\Omega \backslash W) \subset \Omega \backslash W$.

Note that $\Omega_{0}$ is a neighborhood of $W$ in $\Omega$. Let $U$ be a neighborhood of $W$ in $\Omega$ such that $f(U) \subset \Omega_{0}$. Let $g: \Omega_{0} \rightarrow \Omega_{0}$ be an extension of the rotation $R_{2 m \pi} \mid W$ by the maps $\widehat{s}_{j}(w) \mapsto \widehat{s}_{j}(w+2 m \pi)$ (see Proposition 2.11) for a positive integer $m$ large enough to satisfy $g\left(\Omega_{0}\right) \subset U$. Then the composition 
$f \circ g: \Omega_{0} \rightarrow \Omega_{0}$ is well defined and $(f \circ g)(W)=W$. It follows from Property 3.2 that $(f \circ g)\left(\widehat{S}_{0}\right) \subset \widehat{S}_{0}$ and $(f \circ g)\left(\widehat{S}_{1} \cup \widehat{S}_{2}\right) \subset \widehat{S}_{1} \cup \widehat{S}_{2}$. Since $g\left(\widehat{S}_{j}\right) \subset \widehat{S}_{j}$ and $\widehat{S}_{j} \subset S_{j}$ for $j \in\{0,1,2\}$, we have

$$
f\left(S_{0}\right) \cap S_{0} \neq \emptyset, \quad f\left(S_{1}\right) \cap\left(S_{1} \cup S_{2}\right) \neq \emptyset \neq f\left(S_{2}\right) \cap\left(S_{1} \cup S_{2}\right) .
$$

The sets $S_{0}, S_{1}$ and $S_{2}$ are arc components of $\Omega$, and thus $f\left(S_{0}\right) \subset S_{0}$ and $f\left(S_{1} \cup S_{2}\right) \subset S_{1} \cup S_{2}$. Consequently,

$$
f\left(\mathrm{Cl}\left(S_{0}\right)\right) \subset \mathrm{Cl}\left(S_{0}\right), \quad f\left(\mathrm{Cl}\left(S_{1} \cup S_{2}\right)\right) \subset \mathrm{Cl}\left(S_{1} \cup S_{2}\right)=\mathrm{Cl}\left(S_{1}\right) \cup \mathrm{Cl}\left(S_{2}\right) .
$$

Note that $\mathrm{Cl}\left(S_{0}\right) \cap \mathrm{Cl}\left(S_{1}\right)=p_{1} r \cup W$ and $\mathrm{Cl}\left(S_{0}\right) \cap \mathrm{Cl}\left(S_{2}\right)=p_{2} q \cup W$, and $f(\Omega \backslash W) \subset \Omega \backslash W$. Therefore

$$
\begin{aligned}
f\left(p_{1} r \cup p_{2} q\right) & =f\left(\left[\mathrm{Cl}\left(S_{0}\right) \cap\left(\mathrm{Cl}\left(S_{1}\right) \cup \mathrm{Cl}\left(S_{2}\right)\right)\right] \backslash W\right) \\
& \subset f\left(\mathrm{Cl}\left(S_{0}\right) \cap\left(\mathrm{Cl}\left(S_{1}\right) \cup \mathrm{Cl}\left(S_{2}\right)\right)\right) \backslash W \\
& \subset\left[f\left(\mathrm{Cl}\left(S_{0}\right)\right) \cap f\left(\mathrm{Cl}\left(S_{1}\right) \cup \mathrm{Cl}\left(S_{2}\right)\right)\right] \backslash W \\
& \subset\left[\mathrm{Cl}\left(S_{0}\right) \cap\left(\mathrm{Cl}\left(S_{1}\right) \cup \mathrm{Cl}\left(S_{2}\right)\right)\right] \backslash W \subset p_{1} r \cup p_{2} q .
\end{aligned}
$$

Note that $p_{1} r$ and $p_{2} q$ are the only two components of $p_{1} r \cup p_{2} q$. We have two cases.

CASE 1. $f\left(p_{1} r\right) \subset p_{1} r$. The set $p_{1} r$ is an arc, which has the fixed-point property. Hence $p_{1} r$ contains a fixed point of $f$.

CASE 2. $f\left(p_{1} r\right) \subset p_{2} q$. We have $f(r) \in p_{2} q \subset r p_{2}$. Let $h: r p_{2} \rightarrow \mathbb{R}$ be defined by $h(x)=d(x, f(x))$ if $f(x) \in x p_{2}$, and $h(x)=-d(x, f(x))$ otherwise. Thus $h(r)>0$. Note that $h$ is continuous. If $h(x) \geq 0$ for each $x$, then $f\left(r p_{2}\right) \subset r p_{2}$, and thus $r p_{2}$ contains a fixed point of $f$. Otherwise, by the connectedness of $r p_{2}$, there is a point $y \in r p_{2}$ with $h(y)=0$ which is a fixed point of $f$. The proof is complete.

Property 3.4. There exists a disk $D$ in the plane such that $\Omega \cap D$ is an arc (in the boundary of $D$ ) and $\Omega \cup D$ does not have the fixed-point property.

Proof. Let $a b$ be an arc in the plane with $a$ and $b$ as its end points such that $a b \cap \Omega=\{a, b\}$. (In the figure, $a b$ is represented as the straight line segment from $a$ to $b$.) Let $D$ be the union of the simple closed curve $r a \cup a b \cup b r$ and the bounded complementary domain of that curve. To show that $D$ has the desired properties, first define a retraction $g^{\prime}: D \rightarrow r a \cup a b$ and extend it to a retraction $g: \Omega \cup D \rightarrow \Omega_{1}=(\Omega \backslash D) \cup r a \cup a b$ by letting $g(x)=x$ for $x \in \Omega \backslash D$.

Second, define a homeomorphism $h: \Omega_{1} \rightarrow \Omega_{1}$ as follows. For $x \in \widehat{S}_{0} \cup W$ put $h(x)=x$. Also let $h(x)=x$ for $x$ in the arc $K$ from $\widehat{s}_{0}(0)$ to $a$. For $x=\widehat{s}_{j}(w) \in \widehat{S}_{j}$ let $h(x)=\widehat{s}_{2}(w)$ if $j=1$, and $h(x)=\widehat{s}_{1}(w)$ if $j=2$. In other words, using the similarity of the folding patterns of $\widehat{S}_{1}$ and $\widehat{S}_{2}$, these two 
spirals are switched. On the $\operatorname{arc} p_{1} p_{2}$ we define $h$ to be a self-homeomorphism such that $h(a)=a, h\left(p_{1}\right)=p_{2}, h\left(p_{2}\right)=p_{1}, h(r)=q, h(q)=r$.

Further, the homeomorphism $h$, already defined on $p_{1} r \cup p_{2} q$ as a selfhomeomorphism of that set, can also be extended to a self-homeomorphism of $\mathrm{Cl}\left(L_{1}\right) \cup \mathrm{Cl}\left(L_{2}\right)$. Recall that $\mathrm{Cl}\left(L_{1}\right)$ and $\mathrm{Cl}\left(L_{2}\right)$ are both homeomorphic to the $\sin (1 / x)$ curve, and $p_{1} r, p_{2} q$ are their corresponding limit arcs. Extend $h$ to $\mathrm{Cl}\left(L_{1}\right) \cup \mathrm{Cl}\left(L_{2}\right)$ in such a way that $h\left(\mathrm{Cl}\left(L_{1}\right)\right)=\mathrm{Cl}\left(L_{2}\right)$ and $h\left(\mathrm{Cl}\left(L_{2}\right)\right)=$ $\mathrm{Cl}\left(L_{1}\right)$. This completes the definition of $h$. Note that $h(x)=x$ if and only if $x \in K \cup \widehat{S}_{0} \cup W$.

Third, define a map $f: \Omega_{1} \rightarrow \Omega_{1}$ as follows. The map $f$ on $W$ is the rotation $R_{\pi} \mid W$ further extended to the spirals $\widehat{S}_{j}$ by the formula $\widehat{s}_{j}(w) \mapsto$ $\widehat{s}_{j}(w+\pi)$ for $j \in\{0,1,2\}$. Let $z_{1}$ and $z_{2}$ be points in $r a \backslash\{r, a\}$ and $a q \backslash\{a, q\}$, respectively. The map $f$ on $p_{1} r \cup p_{2} q$ is the identity. The arcs $r z_{1}$ and $z_{2} q$ are stretched homeomorphically by $f$ to the arcs $r a$ and $a q$, respectively. In particular, we have $f(r)=r, f\left(z_{1}\right)=a, f(q)=q$, and $f\left(z_{2}\right)=a$. The arcs $z_{1} a$ and $a z_{2}$ are both mapped homeomorphically onto $K$ such that $f(a)=\widehat{s}_{0}(0)$. The arc $K$ is mapped homeomorphically onto $\widehat{s}_{0}([0, \pi])$ with $f\left(\widehat{s}_{0}(0)\right)=\widehat{s}_{0}(\pi)$. The rays $L_{1}$ and $L_{2}$ are homeomorphically mapped onto $L_{1} \cup \widehat{s}_{1}([0, \pi])$ and $L_{2} \cup \widehat{s}_{2}([0, \pi])$, respectively, in such a way that $f\left(\widehat{s}_{1}(0)\right)=\widehat{s}_{1}(\pi), f\left(\widehat{s}_{2}(0)\right)=\widehat{s}_{2}(\pi)$, and $f(x)=x$ for points $x \in L_{1}$ sufficiently near to $p_{1} r$ and $x \in L_{2}$ sufficiently near to $q p_{2}$.

The reader can easily verify that the map $f$ is well defined and continuous, and the composition $f \circ h \circ g: \Omega \cup D \rightarrow \Omega_{1} \subset \Omega \cup D$ is a fixed-point-free map. Therefore $\Omega \cup D$ does not have the fixed-point property.

4. Final remarks and questions. As we mentioned in the introduction, our example is relatively simple. It has only four complementary domains in the plane. It is a countable union of arcs, has only five arc components and one ramification point (of order three). It is natural to ask whether there exist even simpler examples answering Bing's question. The main result of this section states that any such example has to have at least three arc components (see Proposition 4.5 below). To prove this result we need the following three known results.

ObSERVATion 4.1. Let $X$ be a continuum in $\mathbb{R}^{n}$ and $K_{\alpha}$ be a collection of continua in $\mathbb{R}^{n}$ such that $\operatorname{Bd}\left(K_{\alpha}\right) \subset X$ for each $\alpha$. Then $X \cup \cup K_{\alpha}$ is a continuum.

Indeed, this observation follows from the fact that each complementary domain of $X$ is either contained in some $K_{\alpha}$, or disjoint from $\bigcup K_{\alpha}$. Thus the complement of $X \cup \cup K_{\alpha}$ is the union of some of the complementary domains of $X$. This implies that $X \cup \bigcup K_{\alpha}$ is compact. The connectedness of $X \cup \cup K_{\alpha}$ is obvious. 
The next theorem can be proved using the following facts: (i) a locally connected continuum $X \subset \mathbb{R}^{2}$ separates the plane if and only if it contains a simple closed curve that separates the plane between two points in $\mathbb{R}^{2} \backslash X$ ([11, (2.51), p. 107]); (ii) Observation 4.1, and (iii) a locally connected nonseparating plane continuum is an AR ([2, Theorem (13.1), p. 132]) and therefore contractible. The details are left to the reader.

THEOREM 4.2. An arcwise connected planar set $A$ is simply connected if and only if for each disk $D$ in the plane such that $\operatorname{Bd}(D) \subset A$ we have $D \subset A$.

Theorem 4.3 (Hagopian, [3]). Let $X$ be a planar continuum, $f: X \rightarrow X$ be a map, and $\mathcal{P}$ be a partition of $X$ such that $P$ is simply connected and $f(P) \subset P$ for each $P \in \mathcal{P}$. Then $f$ has a fixed point.

COROllary 4.4. Let $X$ be a planar continuum with only finitely many arc components, $A$ be a simply connected subset of $X$ and $f: X \rightarrow A$ be a map. Then $f$ has a fixed point.

Proof. By the continuity of $f$ the set $f(X)$ has only finitely many arc components. Since $A$ is arcwise connected, there is a finite collection of arcs $A_{1}, \ldots, A_{k}$ in $A$ such that $Y=f(X) \cup A_{1} \cup \cdots \cup A_{k}$ is an arcwise connected continuum. Note that for each disk $D$ in the plane such that $\operatorname{Bd}(D) \subset Y$ we have $D \subset A$. Let $U$ be the union of all such disks $D$. Then $Z=Y \cup U \subset A$ and $Z$ is a continuum by Observation 4.1 . Moreover, $Z$ is simply connected by Theorem 4.2. Applying Theorem 4.3 for the map $f \mid Z: Z \rightarrow Z$ and the trivial partition of $Z$ into the only element $Z$, we see that $f$ has a fixed point in $Z$.

Proposition 4.5. Let $X$ be a planar continuum having at most two arc components, and $D$ be a disk in the plane such that $X \cap D$ is an arc. Then $X \cup D$ has the fixed-point property whenever $X$ does.

Proof. Suppose $X$ and $D$ satisfy the hypothesis of the proposition, and assume that $X$ has the fixed-point property. Suppose $f: X \cup D \rightarrow X \cup D$ is a fixed-point-free map. Let $A_{D}$ be the arc component of $X \cup D$ that contains $D$. First we prove that $f\left(A_{D}\right) \subset A_{D}$. Indeed, otherwise $f\left(A_{D}\right) \subset B$ for some arc component $B$ of $X$ such that $A_{D} \cap B=\emptyset$. Let $r: D \rightarrow X \cap D$ be a retraction extended to a retraction $\widehat{r}: X \cup D \rightarrow X$ (by letting $\widehat{r}(x)=x$ for $x \in X)$. Then $\widehat{r} \circ f \mid X: X \rightarrow X$ is a fixed-point-free map, a contradiction.

Since $X$ has the fixed-point property, it cannot be retracted onto a simple closed curve. Therefore, each simple closed curve in $X$ has its bounded complementary domain contained in $X$. This implies that each arc component of $X$ is simply connected by Theorem 4.2. Gluing together $X$ and the disk $D$ along an arc does not change the fundamental group of the arc component of $X$ containing $X \cap D$. Hence the fundamental group of $A_{D}$ has to be 
trivial. Thus each arc component of $X \cup D$ is simply connected. By Corollary 4.4 the image $f(X \cup D)$ cannot be contained in one arc component of $X \cup D$. Therefore $X \cup D$ has an arc component $B$ different from $A_{D}$ and $f(B) \subset B$. Hence $f$ has a fixed point by Theorem 4.3 with $\mathcal{P}=\left\{A_{D}, B\right\}$, a contradiction.

REMARK 4.6. In the plane there exist a continuum $X$ and a disk $D$ such that $X \cap D$ is an arc, $X$ has the fixed-point property and $X \cup D$ does not, and $X$ has only three arc components. The example is more complicated than the continuum $\Omega$ presented in this paper. It has more complementary domains and more ramification points. Therefore it is not included in this paper.

REMARK 4.7. It is natural to ask whether the assumption that the continuum $X$ in Corollary 4.4 has only finitely many arc components can be relaxed. In fact, the authors can prove the following:

Let $f: X \rightarrow A \subset X$ be a map of a planar continuum $X$ into a simply connected subset $A$. Then $f$ has a fixed point.

Our proof, which is quite long, may be included in a future paper.

The continuum $\Omega$ has exactly four complemetary domains in the plane. Planar continua with only one complementary domain, i.e. nonseparating plane continua, are particularly interesting in fixed-point theory. The problem whether all of them have the fixed-point property is almost a century old, remains open, and has been called the most interesting problem in plane topology ([1, p. 122]). Therefore, we ask the following natural question.

QUESTION 2. Let $X$ be a nonseparating plane continuum with the fixedpoint property, and $D$ be a disk in the plane such that $X \cap D$ is an arc. Must $X \cup D$ have the fixed-point property? What if $X$ has exactly two (three) complementary domains?

Let $X$ be a continuum with the fixed-point property. In the past the fixed-point problem for the unions of $X$ with disks (as in Questions 1 and 2) has been associated with the problem for the cylinder over $X$, i.e. the product $X \times[0,1]$. The motivation for the product problem is similar to the one for unions (see the discussion in the introduction) and comes from the fact that the product of two AR's is an AR (see [2, Theorem 7.1, p. 92]). The cylinder over each of the examples by Knill [6] and Lopez [7] mentioned in the introduction does not have the fixed-point property and is a counterexample for products. W. L. Young [13] showed that the cylinder over Bing's continuum ([1, Theorem 14]) has the fixed-point property. Bing asked the following question. 
Question 3 (Bing, [1, Question 9]). If $C$ is a 1-dimensional continuum with the fixed-point property, must $C \times[0,1]$ have the fixed-point property?

Question 3 remained open for over thirty years until recently M. Sobolewski answered it in the negative with his example [10]. It is still unknown whether there exists such an example in the plane.

Question 4 (Bing, [1, Question 10]). If $C$ is a continuum in the plane with the fixed-point property, must $C \times[0,1]$ have the fixed-point property?

The properties of $\Omega$ established here can be used to prove that the cylinder $\Omega \times[0,1]$ has the fixed-point property. Question 4 remains a major challenge in the area.

\section{References}

[1] R. H. Bing, The elusive fixed point property, Amer. Math. Monthly 76 (1969), 119132.

[2] K. Borsuk, Theory of Retracts, Monograf. Mat. 44, PWN-Polish Sci. Publ., Warszawa, 1967.

[3] C. L. Hagopian, The fixed-point property for simply connected plane continua, Trans. Amer. Math. Soc. 348 (1996), 4525-4548.

[4] C. L. Hagopian and R. Mańka, Simple spirals on double Warsaw circles, Topology Appl. 128 (2003), 93-101.

[5] V. Klee and S. Wagon, Old and new unsolved problems in plane geometry and number theory, Dolciani Math. Exp. 11, Math. Assoc. America, Washington, DC, 1991.

[6] R. J. Knill, Cones, products and fixed points, Fund. Math. 60 (1967), 35-46.

[7] W. Lopez, An example in the fixed point theory of polyhedra, Bull. Amer. Math. Soc. 73 (1967), 922-924.

[8] R. Mańka, Association and fixed points, Fund. Math. 91 (1976), 105-121.

[9] —, On spirals and fixed point property, ibid. 144 (1994), 1-9.

[10] M. Sobolewski, A curve with the fixed point property whose cylinder admits a fixed point free map, Houston J. Math. 31 (2005), 239-253.

[11] G. T. Whyburn, Analytic Topology, rev. ed., Amer. Math. Soc. Colloq. Publ. 28, Amer. Math. Soc., Providence, RI, 1963.

[12] G. S. Young, Fixed-point theorems for arcwise connected continua, Proc. Amer. Math. Soc. 11 (1960), 880-884.

[13] W. L. Young, A product space with the fixed point property, ibid. 25 (1970), 313-317.

Department of Mathematics

California State University, Sacramento

Sacramento, CA 95819-6051, U.S.A.

E-mail: hagopian@csus.edu

prajs@csus.edu
Institute of Mathematics and Informatics

Opole University

Oleska 48, Opole, Poland 